\title{
Perfil Estructural - Sistémico Familiar en el Hospital Hermilio Valdizán
}

\author{
ALFONSO MENDOZA, JOSE GALINDO, LIZARDO RODRIGUEZ, LALRA OSSO. IDA ALFARO, \\ MARIA MLRGADO. WILFREDO MORMONTOY. \\ Departamento de la Familia " Sistemas IHmanos del Mospital Hermilio Valdizan \\ Sede Docente de la Faculad de Medicina. Universidad Nacional Mayor do San Marcos
}

\begin{abstract}
RTSLMTN
En el presente trabajo se estudia el Perfil Fstructural Sistémico de veinte familias nucleares completas que liene a uno de sus miembros como paciente nuevo en la consulta externa de un hospital psiquiátrico estatal. Como instrumento de medición se utilixa la Fscala Familiar Estructural Sistémica, que evalúa a la familia en cinco dimensiones: Estructura, Estadio del Desarrollo, Resonancia, Etiquetación det Paciente Identificado y Resolución de Conflictos. Los resultados muestran una significativa disfuncionalidad global, así como en las dimensiones Resolución de Conllictos, Eliquetación del Paciente Identificado y Fstructura. Se discute estos resultados y se les compara con estudios de otros autores.
\end{abstract}

Palahras Claves: Perfil de Salud, Enfoque Sistémico, Evaluación Familiar, Terapia Familiar.

\begin{abstract}
SYSTEMIIC-STRUCTLRAL NUCI,EAR FAMILY PROFILE, OF FAYILIES AT HERMILIOVALDIZAN HOSPITAL SLIMMARY

This paper studies the Structural Family System Profile of twenty nuclear families, in which one nuclear member is a new outpatient of a public psychiatric hospital. Structural Family System Ruting is used as an instrument to asses the family in five dimensions: structure, developmental stage, resonance, identified patienhorid and conflict resolution. The results show a global significant disfunctionality, especially in conflict resolution, identified patienthood and struclure. We compare these results with others studies.
\end{abstract}

Key' Words: Heallh Planning, Systems Management, Family Therapy, Family Evaluation.

\section{INTRODUCCIÓN}

Las evaluacianes familiares tienen una historia que, de acuerdo con Tseng ('), puede remontarse a Ackeman y Bchrens (1956), quienes consideraron Ios aspecios psicosociales de las familias; también a Voiland y Buell (196!), quienes se centraron en la psicopatología de los padres; a Goldstein (1968), quien incento clasificar a las familias de acuerdo con los problcmas do Ios adolescentes; a Wertheim (1973). quicn usó las tres ưimensiones de «.Morfogénesis» inducida (crecimiento y cambio). "Morfostasis» consensual (estabilidad y solidaridad) y "Morfostasis» forzada; a Ritcher (1974), quien trabajó los conceptos de «síntoma de ncurosis familiar» y «carácter ncurótico familiars; y a otros, como Fisher (1977), quicn hizo una exicnsa revisión de varios sistemas de clasificación, y al mismo Tseng (') quien Irabajo la clasificación Iriaxial.

\footnotetext{
Correspondencia:

Dr. Alfonso Menduza Fermandez

Facultad de Medicina U. N.M.S.M.

Av Ciran 755. Lima I - Perí
}

En los años ochentas diversos investigadores se abocaron a consıruir instrumentos que. desde la perspectiva sistémica, hicieran posible la evaluación de las familias, observándolas. sea en condiciones experimentales, en cuyo caso se evalúa la reacción del sistema familiar frente a tareas o problemas de cierta complejidad, sea err el marco de las situaciones corrientes de la vida. En esia úlíma línea, tres son los modelos que cabe resaltar: el modelo circunflejo de Olson, la escala de BeaversTimberlawn y el modelo Mc. Master, de Epstcin.

Para Fontaine (⿳亠丷厂 ), la escalii de autoevaluación de Olson y col. es la mejor consiruida desde los puntes de vista conceptual y cstadístico y, de hocho, ha resultido la más ampliamente ulilirada en la investigación clínica.

En nucstro pás son escasos los trabajos de investigación sobre lamilia desde la perepectiva sistémica y. mas aún, los referentes a evaluación familiar. En este campo, el trabajo de Castro de la Malla ( ${ }^{3}$ ) marcó un precedenic, y es a partir de 1980 que profesionales de la salud monal llevaron a cabo investigaciones sobre la familia desde esta óptica. Mencionemos a Zevallos (1985), quien aplicó la Escala de Sobreproteccióll y 
Sobreindulgencia de Rosen en la relación madre-hijo farmacodependiente; y. aplicando los cuestionarios Faces Ir y III de la Escala de Olson, a Malamud ( $\left.{ }^{4}\right)$, Francis ("), Cañavera (i), Quintana (), Bernós $\left({ }^{8}\right)$, Ráez $\left({ }^{9}\right)$ y Quiroz $\left({ }^{10}\right)$.

En nuestro estudio hemos intenlado encontrar un perfi] diagnóstico (estructural-sistémico) de familias atendidas en una inslitución de salud mental, que nos proporcione información consistente acerca de las características más relevantes de familias peruanas que lienen uno de sus miembros afectado por un trastorno psiquiátrico (paciente identificado, $P I$ ). Sin embargo, señalamos que una importante limitación en este trabajo estuvo dada por el tamaño de la muestra, que no ha permitido realizar generalizaciones con el rigor estadístico requerido.

Esta investigación, que se sitúa en la línea de la evaluación en condiciones experimentales, ha sido realizada urilizando el instrumento desarrollado por Szapocznik y cols. ('1), a partir de] enfoque estructural de Minuchin $\left({ }^{12-15}\right)$. Tal instrumento, en su versión de 1991, apunta a encontrar valores cuantificables en las 5 dimensiones siguicntes: Estructura, que mide los patrones de interacción familiar referidos a liderazgo, organización de los subsistemas y flujo de la comunicación; Resonancia, que mide la diferenciación entre los subsistemas tomando en cuenta los umbrales de sensibilidad de cada uno de los miembros hacia los otros (involucramiento y desinvolucramiento, ambos con extremos disfuncionales); Estadio del Desarrollo, que compara el momento cronológico del ciclo vital de la familia con las características de la interacción dentro de los diferentes subsistemas: Esiqueración del Paciente Identificado, que evalúa en qué medida la familia escá convencida que el problema primordial es sólo la patología del paciente; Resoluciön de Confícios, que mide la habilidad del sistema familiar para expresar. confrontar y negociar diferencias de opinión y desacuerdos.

El proceso de oblención de datos estadísticos nos ha permitido validar el instrumento y apreciar su utilidad en tanto pueda ser aplicado a contexios clínicos más amplios y a otros no clínicos.

\section{MATERIAL Y MÉTODOS}

Se trabajó con veinte familias nucleares completas que tuvieron, por presentar algún trastorno psiquiátrico, a uno de sus miembros como paciente nuevo en la consulta externa del Departamento de Salud Mental de Adultos y Geriatría del Hospital Hermilio Valdizán. Para alcanzar el tamaño de lá muestra fue necesario citar a 79 familias; dicha cila se realizó el día de la primera consulta del PI, donde se explicó a los miembros presentes sobre el proceso de evaluación, se recabó la autorización escrita de su participación y se les recomendó que asistieran ambos padres, el PI y el mayor número de los otros miembros, si no fuera posible que acudieran todos. De las familias convocadas, 36 desertaron, 5 fueron descarladas por presentar problemas técnicos en el nraterial videograbado y 18 por tratarse de familias extensas o incompletas.
El discño del escudio fue transversal, prospccivo y descriptivo. A todas las familias se les evaluó mediante la Escala Familiar Estructural Sistémica (EFES), que es un instrumento complejo de observación estructurada, en la que se registra la aparición y/o la frecuencia de conductas especificadas de antemano en una lista de confrontación. Está consta de dos partes:

$1^{\circ}$ Un juego de estímulos estandarizados, llamado Tareas Familiares, diseñado para someter a la fámilia a situaciones crecientes de estrés, para luego observar los patrones interaccionales (operacionalizados) de la tamilia a lo largo de las siguientes dimensiones: Estrucrura, Resonancia, Estadio del Desarrollo, Eriquetación del Paciente Identificado y: Resolución de Conflicios.

$2^{\circ}$ Un jucgo de 5 escalas, elabojado especialınente para evaluar clínicamente y calificar las respuesias videograbadas de las tamilias en las dimensiones ya señaladas.

La aplicación del instrumento fue realizada en 3 elapas:

a. Se propuso, a través de una cinta grabada, las instrucciones de 3 siluaciones diferentes de ejercicios en vivo (Tareas Familiares), donde la familia fue animada a actuar lo más naturalmente posible en una sála acondicionada para tal fin, sin la intervención direcla de los investigadores: quienes se situaron fuera de este ambiente. Enl la tarea I se les solicitó que escogieran un menú para la cena que les guste a todos, el cual debería contener un tipo de carne, dos vegetales, una bebida y un postre; en la tarea II se les pidió que cada uno de los miembros verbalizará lo que les gusla y les disgusia de las cosas que hacen en casa; y en la carea III que abordaran un problema familiar ocurrido en casa, precisando quiénes parliciparon, como se originó y cómo terminó dicha situación. Cada una de las lareas tuvo una duración de 5 minulos. adicionándosele (para las tareas II y III) 1 minuto por cada miembro que excediera al número de 5, hasta un máximo de 10 minutos. La ejecución de las lareas se videograbó para ser calificada posteriormente.

b. En un segundo momento, 5 miembros del equipo de investigación entrenados en la EFES, calificaron en forma conjunca utilizando las respuestas videograbadas y la transcripción de las interacciones verbales producidas durante las tres tareas.

c. Las calificaciones fueron transformadas en resultados finales (Perfil Estructural-Sistémico) con ayuda del Programa de Calificación de la EFES, elaborado específicamente para cuantificar las dimensiones investigadas.

Al finalizar la calificación, cada una de las dimensiones tuvo un puntaje de 1 a 5 , que mide de menor a mayor el nivel de funcionalidad; además de uis Puntaje Toral (PT), que mide el nivel de funcionalidad global de la familia y cuyo valor puede variar de 5 a 25 . Con la finalidad de poder comparar este últino resultado con las dimensiones anteriores se obtuvo el Puntaje Total Estratificado (PTE), en el que consideramos Nivel 1 (PT de 
5 a 9), Nivel 2 (de 10 a 13), Nivel 3 (de 14 a 17), Nivel 4 (de 18 a 21 ) $y$ Nivel 5 (de 22 a 25).

Los resultados fueron agrupados en lablas de frecuencias absolulas y relativas (\%), así como en gráfico de barras y de tendencias. En cada una de las dimensiones se halló la mediana y los cuartiles l y 3. Para evaluar la significación estadística de las tendencias se aplicó la prueba binomial, tomando como punto de corte la Mediana Térica (nivel 3), descamando los valores iguales a dicha mediana s comparando los niveles $1+2$ con los niveles $4+5\left({ }^{16}\right)$

\section{RESULTADOS}

Las 20 familias seleccionadas, que cumplieron con los criterios de inclusión -familia nuclear completa con un paciente nuevo atendido en la Consulta Externa del Hospilal- presentaron las siguicntes características demográficas:

- Estado civil de las parejas: casados, 17 familias $(85 \%) ;$ y convivientes, 3 familias (15\%).

- Familias reconstituidas: 3 (15\%) y, el resto, 17 (85\%), correspondió al primer compromiso de ambos miembros de la pareja.

- Número promedio de hijos: 4.0 (límites de 1-12). cuyas edades oscilaron entre 3 y 37 años (promedio 17,5).

- Edad promedio de los padres: 46 años, y de las madres 41,2 años; siendo el promedio de la diferencia de estas edades de 5,7 años.

- Grado de instrucción del padre: superior complela. $5(25 \%)$; superior incompleta, 2 (10\%); secundaria completa, 3 (15\%); secundaria incomplela, 3 (15\%); primaria completa. $6(30 \%)$; primaria incompleta, $1(5 \%)$ y analfabetos, $0(0 \%)$.

- Grado de instrucción de la madre: superior completa, $3(15 \%)$; superior incompleta, 1 (5\%); sccundaria completa, $3(15 \%)$; secundaria incomplcla, 4 (20\%); primaria complcta. 5 (25\%); primaria incompleta, 2 (10\%) y analfabetas, 2 (10\%).

- Grado de instrucción de los hijos adultos (mayores de 18 años): superior completa, $18(41.9 \%)$; superior incomplcta. $10(23.3 \%)$; secundaria completa, $10(23.3 \%)$ y secundaria incompleta, $5(11.6 \%)$

- Procedencia de las familias: de Lima y Callao, 15 (75\%) y de provincias, $5(25 \%)$.

- Ubicación geográlica de las familias: zona rural, 2 (10\%): zona urbano-marginal, $6(30 \%)$ y zona urbana, $12(60 \%)$.

- Paciente identificado: en 9 familias (45\%). se ubic6 en el subsisicma parental; y en 11 familias $(55 \%)$, en cl subsistema filial. De las 9 primeras familias, el padre fue el afectado en 6 casos $(66,7 \%)$ y la madre en los olros $3(33,3 \%)$.

Aplicando el instrumento de evaluación se halló el Pcrfil Estructural Sistémico de cada una de las 20 familias.

En la dimensión Esrructura se halló que 1 familia (5\%) se encontraba en el Nivel I(muy disfuncional). 9 familias (45\%) en el Nivel 2 (disfuncional), 7 familias (35\%) en el Nivel 3 (promedio), 2 familias (10\%) en el Nivel 4 (bueno) y 1 familia (5\%) en el Nivel 5 (excelente). Mediana: $2,5(\mathrm{Q}=2, \mathrm{Q} 3=3):(\mathrm{p}<0.05)$ (Fig. $\mathrm{N}^{0}$ 1).

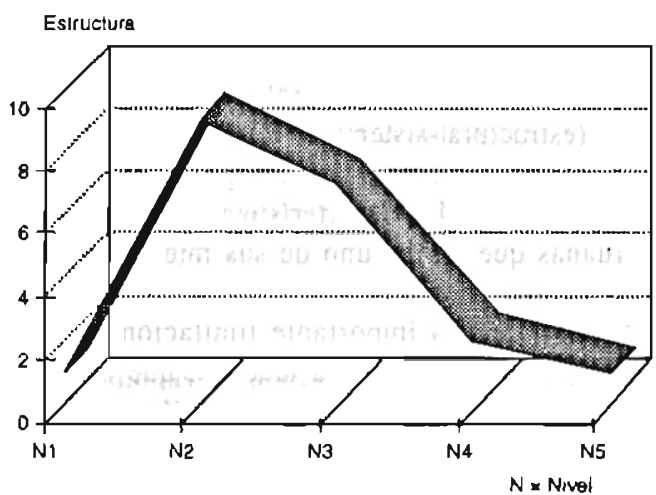

Fig. 1.- Distribución de Frecuencias. Dimensión Estructura $(p<0,05)$.

En la dimensión Estadio del Desarrollo se halló que 2 familias (10\%) alcanzaron el Nivel 1 (muy disfuncional), 6 familias (30\%) el Vivel 2 (disfuncional), 9 familias (45\%) el Nivel 3 (desarrollo un tanto atenuado). 3 ramilias (15\%) el Nivel 4 (bueno), y ninguna el Nivel 5 (excelente). Mediana: $3(\mathrm{Q} 1=2, \mathrm{Q} 3=3)$; $\left(\mathrm{p}=0,113\right.$ no significativo) (Fig. $\mathrm{N}^{\circ} 2$ ).

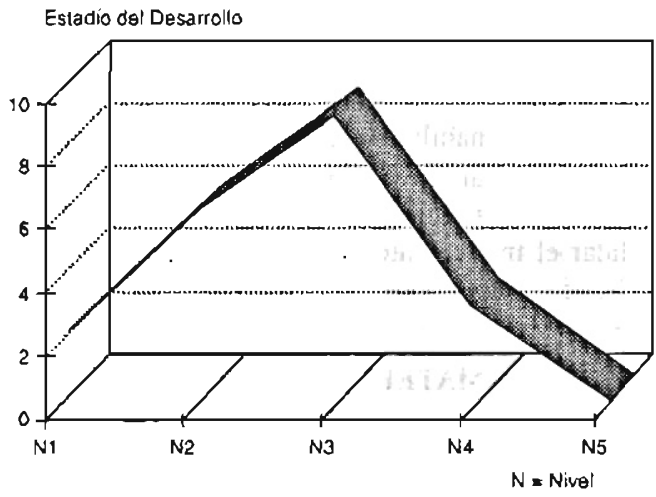

Fig. 2.- Distribución de Frecuencias. Dimensión Estadío del desarrollo $(p<0,113)$

En la dimensión Resonancia se halló que 2 familias (10\%) alcanzaron el Nivel 1 (límites impermeables o inexistentes), 5 familias $(25 \%)$ el Nivel 2 (límites poco permeables o no definidos), 7 familias ( $35 \%$ ) el Nivel 3 (límites un tanto permeables o un tanto definidos), 6 familias (30\%) el Nivel 4 (límites permeables y moderadamente definidos), y ninguna el Nivel 5 (límites permeables y bien definidos). Mediana: $3(\mathrm{Q} l=2$ y $\mathrm{Q} 3=4)$ $p=0.500$ (no significativo) (Fige $\mathrm{N}^{\circ} 3$ ). 


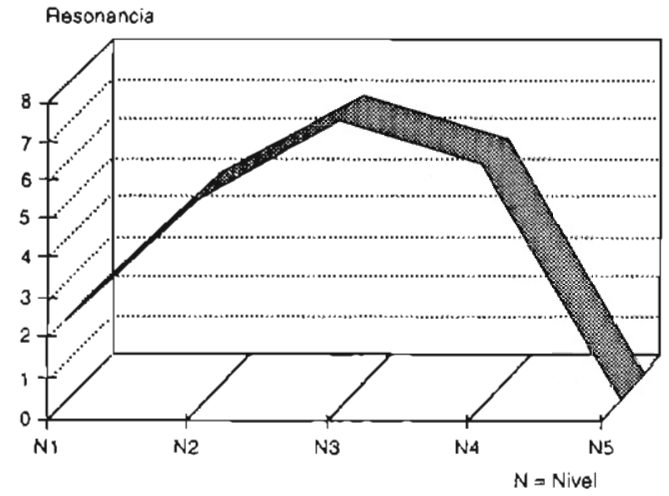

Fig. 3.- Distribución de Frecuencias. Dimensión Resonancia $(\mathrm{p}<0,500)$.

En la dimensión Efiquetación del Paciente Identificado se hallo que 3 familias (15\%) se enconitraban en el Nivel 1 (etiquetación muy rígida), 15 familias (75\%)en el Nivel 2 (etiquetación moderadamente rígida), ninguna familia en el $\mathrm{Ni}$ vel 3 (etiquetación un tanto flexible), 1 familia (5\%) en el Nivel 4 (etiquetación moderadamente flexible), y 1 familia (5\%) en el Nivel 5 (etiquetación muy flexible). Mediana: $2(\mathrm{Q} 1=2$ y Q3= 2); $p<0,001$ (altamente significativo) (Fig. $N^{\circ} 4$ ).

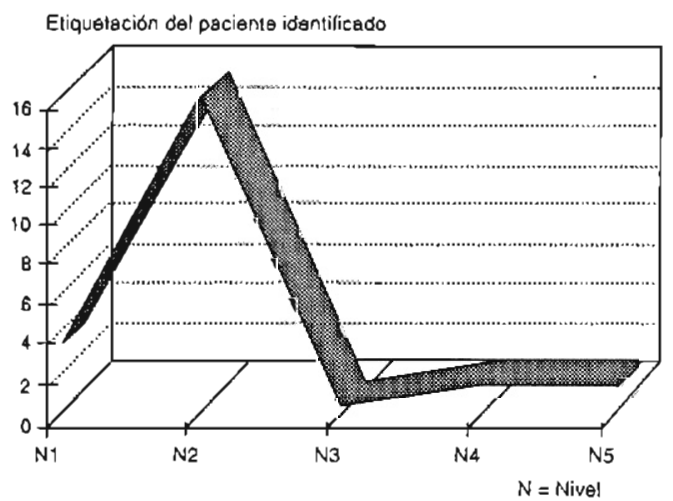

Fig. 4.- Distribución de Frecuencias. Dimensión Etiquetación del Paciente Identificado $(p<0,0001)$

En la dimensión Resolución de Conflictos se balló que 19 familias (95\%) alcanzaron el Nivel 1 (muy pobre capacidad de resolución), 1 familia (5\%) el Nivel 2 (pobre capacidad de resolución) y ninguna familia alcanzó el Nivel 3 (mediana capacidad de resolución), el Nivel 4 (buena capacidad de resolución), ni el Nivel 5 (excelente capacidad). Mediana: $\mathrm{l}(\mathrm{Q} \mathrm{l}=1$ y $\mathrm{Q} 3=1)$; $\mathrm{p}<0,001$ (altamente significativo) (Fig $\mathrm{N}^{\circ}$ 5).

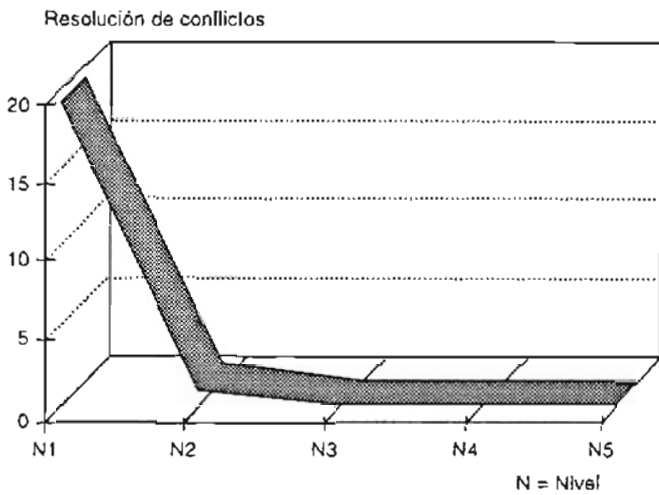

Fig. 5.- Distribución de Frecuencias.

Dimensión Resolución de conflictos $(p<0,0001)$

El Punraje Toral fue $7 \mathrm{cn} 1$ familia (5\%), 9 puntos en 2 familias (10\%), 10 puntos en 4 familias $(20 \%), 11$ puntos en 4 familias $(20 \%), 12$ puntos en 3 ramilias ( $15 \%$ ), 13 puntos en 5 familias $(25 \%)$ y 16 puntos en 1 familia $(5 \%)$. La mediana fue de $11(\mathrm{Q}=10$ y $\mathrm{Q} 3=13)$.

El Puntaje Total Estratificado distribuyó a 3 familias (15\%) en el Nivel 1 (muy disfuncional). 16 familias ( $80 \%$ ) en el Nivel 2 (disfuncional), 1 familia ( $5 \%$ ) en el Nivel 3 (funcionalidad mediana) y ninguna en los Niveles 4 (buena funcionalidad) ni 5 (exceIente funcionalidad). La mediana fuc de $2(\mathrm{Q} \mid=2$ y $\mathrm{Q} 3=2) ; \mathrm{p}<0,001$ (altamente significativo).

\section{DISCUSIÓN}

En el presente rabajo se ha estudiado el perfil estructural sistémico de veince familias nucleares completas, analizando comparativamente cada una de las dimensiones familiares.

Los resultados en la dimensión Estructura presentaron una tendencia estadísticamente significativa hacia la disfuncionalidad, lo cual nos hace pensar que en estas familias existen deficiencias en su organización interna, es decir, probiemas en la jerarquía, en el modo de controlar los comportamientos inadecuados de los miembros subordinados, en la capacidad de guiar adecuadamente a la familia, así como en el ejercicio democrálico de la autoridad. Esle resultado reflejaría cambién la presencia de estilos comunicacionales inadecuados, lales como triangulaciones (interferencia de un miembro de la familia en un conflicto entre otros dos) e incapacidad para establecer comunicaciones claras y directas, entre otros. No obstante, no se halló grados extremos de disfuncionalidad ( 9 familias en nivel 2 y solamente I familia en el nivel I), lo que se explicaria, tal vez, por estar constituida la muestra por familias nucleares completas, las cuales tienden a presentar una organización más apropiada que las familias incompletas. 
La dimension Resonancia presentó una distribución francamente central (no disfuncional). Dado que no existen en nucstro país investigaciones (utilizando la EFES) con familias sin un PI, hemos supuesto que la curva de distribución (desde el punto de vista estadístico) deba ser normal y tener una mediana teórica en el nivel 3. Consideramos también que estudios con familias que no posean un paciente identificado podrían arrojar resultados con tendencia hacia la funcionalidad (niveles 4 o 5) y la comparación de nuestros resultados con aquellos nos mues. Ire diferencias con mayor significación estadística. Refuerzan esta última hipótesis los estudios realizados por Quintana () en una población correspondiente a un estrato socioeconómico bajo de Lima Metropolitana, y por Quiroz $\left({ }^{10}\right)$ en olra de un estrato medio del Cusco, quienes (aplicando el cucstionario Faces II) encontraran una alta incidencia de familias que se situaron en un nivel adecuado en el eje de cohesión emocional. lo que sería equivalente a los niveles funcionales de la EFES.

La ausencia de disfuncionalidad en la dimensión Reronancia podría ser explicada, en parle, por la ausencia de disfuncionalidad en la subdimensión Involucramiento/ Aglutinamiento, la misma que ha sido relacionada principalmente -aunque no exclusivamente-con la aparición de trastornos psicosomáticos, los cuales son atendidos, mas bien, en hospitales generales y no en instituciones psiquiátricas como la nuesira.

La dimensión Estadio del Desarrollo presento resultados con una distribución central (no disfuncional), hallazgo que estaría en discordancia con las observaciones clínicas de algunos teóricos de la Terapia Familiar ( ${ }^{17 \cdot 211}$ ), quienes relieren la aparición de un PI en contextos familiares donde se encuentra severas dificultades para que los hijos logren la independización. Sin embargo, al estudiar el subgrupo de la muestra que incluye a las familias donde el PI pertenece al subsistema filial (11 familias), encontramos una tendencia estadísticamente significativa a la disfuncionalidad $(p<0,05)$, conservando su tendencia central el subgrupo de familias donde el PI es uno de los padres, lo cual concuerda con la hipótesis mencionada.

A pesar de proceder las familias de nuestro estudio de un contexto sociocultural donde obscrvamos que la separación de los hijos tiende a realizarse tardíamente y en algunos casos no se realiza, los resultados presentaron una tendencia no disfuncional en las dimensiones Resonancia y Esradio del Desarrollo, lo que nos hace pensar que las familias nucleares completas, con las que hemos trabajado, poseen mayores recursos para atravesar las diferentes etapas del ciclo vital que otro tipo de familias. Dilucidar este punto requeriría de investigaciones que incluyan a familias extendidas incompletas y otras.

La tendencia hacia la «no disfuncionalidad» en las dimensiones Estadio del Desarrollo y Resonancia, también, puede hacernos suponer que éstas sean variables poco sensibles (en nuestro medio) a la presencia de un PI dentro de la familia.
En las dimensiones Etiquetación del Paciente Identifica. do $y$ Resolución de Conflicios encontramos una franca tendencia a la disfuncionalidad, a predominio de la última. Pensamos que la característica que identificó a nuestra muestra, esto es, el de tener en su seno a un PI, explicaría el alto nivel de disfuncionalidad en la primera dimensión arriba citada, en la cual observamos una elevada tendencia a señalar al paciente como el supuesto origen de sus dificultades $y$, en algunos casos, a considerarlo como la única causa de sus problemas.

Observamos también una concordancia entre los resultados de la dimensión Etiquetación del Paciente Identificado y el Puntaje Total Estratificado. Este dato confirmaría una de las premisas básicas del enfoque sistémico de la Terapia Familiar, el cual postula que las familias rígidas tienden a organizarse en función del síntoma y que éste, a su vez, cumple una función reguladora dentro de la homeostasis familiar.

La baja capacidad de resolución de conflictos mostrada por nuestras familias concordaría con las investigaciones realizadas utilizando otros insirumentos, como el Cuestionario Faces II de Olson $\left(^{4-k}\right)$, en las cuales los bajos niveles de adaptabilidad publicados coincidirían con los bajos resultados hallados por nosotros en esta última dinensión. Es decir, encontramos una escasa capacidad para resolver las dificultades, ya sea evitando que emerjan los conflictos, represión; o interrumpiéndo. los abruptamente luego de su aparición, evasión.

En el Puntaje Total Estratificado, que relleja el perfil global de la familia, 16 se ubicaron en el rivel 2 (disfuncional), el cual no corresponde al nivel más bajo de la escala. Dado que nuestro hospital aticnde a los sectores socioeconómicos menos favorccidos, esperábamos encontrar mayor cantidad de familias en cl Nivel l (muy disfuncional); sin embargo, solamente hallamos 3. Esto podría tener dos explicaciones, primero, que las fanilias con un perfil muy disfuncional no han desarrollado la capacidad de organización más elemental que los lleve a solicitar ajuda a una institución de salud; $y$, segundo, que la crisis económica de nuestro pais ha determinado que nuestro hospital atienda cada vez más a poblaciones de estratos socioeconómicos medio y aún medio allo, desplazando a las de estratos bajos.

En vista de que sólo una familia alcan $\angle 6$ el nivel 3 (inlêrmedio) y ninguna niveles superiores (buena y excelente funcionalidad), podemos aseverar que codas las familias con un $\mathrm{Pa}$ ciente Identificado $(P I)$ mostraron un perfil de funcionamiento pobre. Estudios con muestras más amplias y diversificadas permitirían analizar mejor el comportamiento de esta variable, asi como comparar a las familias que tengan un miembro con patología psiquiátrica con aquellas que no lo tengan.

El bajo nivel de funcionamiento de la familia, que se objetiva en un Puntaje Total Estratificudo francamente disfuncional, podría estar relacionado con la presencia de comportamientos calalogados como trastornos psiquíátricos; resultado similar al 
que comunica Szapocznik $\left(^{11,21.22 .23}\right)$ estudiando familias con miembros que presentaban comportamientos delincuenciales y de adicción a sustancias psicoactivas.

\section{CONCLUSIONES}

1. La EFES es un instrumento de evaluación familiar útil para calificar cuantitativamente el funcionamiento familiar.

2. Las familias estudiadas mostraron una disfuncionalidad global significativa.

3. Dentro del Perfil Estructural Sistémico determinado en la muestra, se encontró disfuncionalidad significativa en las dimensiones Resolución de Conflictos, Etiquetación del Paciente Identificado y Estructura, siendo mayor en la primera.

4. La disfuncionalidad en la dimensión Estadío del Desarrollo estuvo asociada con la presencia del PI en el subsistema filial, no así cuando él pertenecía al subsisterna parental.

5. Existe una asociación estadísticamente significativa entre la dimensión Etiquetación del Paciente Identificado y el nivel de funcionalidad global de la familia.

\section{AGRADECIMIENTOS}

A los Dres. José Szapocznik, Olga Hervis y Victoria Mitrani, del Spanish Family Guidance Center, Departament of Psychiatry, University of Miami, por su asesorfa permanente y autorización para la utilización del instrumento (SFSR).

A la Dra. Raquel Cohen, por su constante estímulo.

Al Dr. Elard Sánchez, Director Ejecutivo del Hospital Hermilio Valdizán, y al personal en general por el apoyo institucional al presente trabajo.

A los laboratorios CIBA-GEIGY Lima, Perú, por facilitarnos el uso de sus ambientes como recintos de filmación y sus equipos de videograbación en gran parte del trabajo.

A la Dra. Margarita Yactayo, por su participación en las etapas iniciales del proyecto.

A las familias que gentilmente accedieron a participar en la evaluación.

\section{BIBLIOGRAFIA}

1) Tseng, W.S.; Mc Dermoll. J.F.- "Triaxial Family Classification». Journal of Child Psychiatry. Vol 18, $\mathrm{N}^{\circ}$ 1.- Yale University Press. New Haven and London.1979

2) Fontaine, P.- «Evaluation de familles: Modèles es Échelles». Dictionaire Clinique des Thérapies Familiales Systémiques, p. 189. 196. Les Éditions ESF, Paris. 1988

3) Castro de la Matta, R.- «Un intento de Clasificación de la Familia Peruanay. Tesis Doctoral. Programa Académico de Medicina, Universidad Peruana Cayetano Heredia. Lima-Perú, 1972

4) Malamud, F.- *Un modelo para la descripción familiar: Aplicación del Faces II en el cono norte de Limax. Tesis de Bachiller, Pontificia Universidad Católica del Perú. Inédita. 1987
5) Francis, C.- «La Satisfacción Familiar como criterio de evaluación». Tesis de Bachiller. Pontificia Universidad Católica del Perú. Inédito. 1987

6) Cañavera, M.- «Diada Marital Disfuncional y Farmacodependencia a Pasta Básica de Cocainam. Tesis de Bachiller, Pontificia Universidad Carólica del Perú. Inédita. 1988

7) Quintana, A.- aReconstitución Familiar por Línea Materna: Ca. racterísticas y Funcionalidad en un Sector Socioeconómico Bajo de Lima Metropolitanaw. Tesis de Bachiller, Pontificia Universidad Calólica del Perú. Inédito. 1990

8) Bernós, R. «Esquizofrenia y Disfuncionalidad Familiarm. Tesis de Bachiller, Pontificia Universidad Calólica del Perú. Inédito. 1990

9) Ráez, M. - «Fanilia y Adolescencia: Tipos de Familia y Modelo Ideal Familiar, Aplicación del Cuestionario Faccs lll en un sector socioeconómico de Liman. Tesis de Licencialura. Universidad Femenina del Sagrado Corazón. Lima Perú. Inédito. 1992

10) Quiroz, R; Mendoza, A; Holgado, M; Quintana, A.- «Tipologín de la familia cusqueña de acuerdo al modelo circunflejo de Olson". Trabajo presentado al XIIl Congreso Nacional de Psiquiatría. LimaPerú. Inédilo. 1994

11) Szapocznic J.; Río, A.; Hervis, O.; Bchar, V.; Kurtines, W.; Faraci, A.- "Assessing Change in Fantily Functioning as a Result of Treatment: The Structural Family System Rating Scale (SFSR)w. Journal of Marital and Family Therapy. Vol. 17, $\mathrm{N}^{\circ}$ 3.- Miami-USA. 1991.

12) Minuchin, S; Fishman, H.- "The Psychosomatic Family in Child Psychiatry». Journal of Child Psychiatry. Vol 18. No 1.- Yale Universicy Press. New Haven and London. 1979

13) Minucbin, S.- «Familias y Terapia Familiar». Editorial Gedisa. Tercera Edición. Buenos Aires-Argentina. 1982

14) Minuchín, S.- *Técnicas de Terapia Familiar». Editorial Paidós. Buenos Aires-Argentina. 1984

15) Minuchin, S.- "Caleidoscopio Familiar». Editorial Paidós. Buenos Aires-Argentina. 1985

16) Schefler, W.- «Bioestadistica». Fondo Educativo Interamericano S.A. Estados Unidos de América. 1981

17) Haley, J.- «Terapia para Resolver Problemas». Editorial Amorroriu. Buenos Aires-Argentina. 1980

18) Haley, J.- “Terapia Na Convencional». Edicorial Amorrortu. Bue. nos Aires- Argentina. 1985

19) Haley, J.- «Trastornos de la Emancipación Juvenil y Terapia Familiar». Editorial Amorrortu. Buenos Aires-Argentina. 1985

20) Stanton, M.D.; Todd, T.C. y cols.- «Terapia Familiar del Abuso y Adicción a las Drogas». Editorial Gedisa. Barcelona-España. 1988

21) Szapocznic J.y col.- «Bceakthrougths in Family Therapy with Drug-Abusing and Problem Youth*. Springer Publishing Company, New York. 1989

22) Szapoeznic J.; Santisteban, D.; Río, A.; Perez-Vidal, A.; Santisteban, D; Kurtines, W. - «Family Effectiveness Training: An Intervention to Prevent Drug Abuse and Problem Behaviors in Hispanic Adolescent». Hispanic Journal of Behavioral Sciences. Vol. 11. $N^{\circ}$ 1. pp 4-27.- Miami-USA. Feb. 1989

23) Hervis, O.; Szapocznik, J.; Behar, V.; Rio, A.; Kurtines, W:. "Structural Family Systems Ratings: A Revised Manual». Spanish Family Guidance Center Departament of Psychiatry. School of Medicine. University of Miami. Nov. 1991 